\title{
Spatial Reasoning Ability and the Construction of Integrals in Physics
}

\author{
Nathaniel R. Amos and Andrew F. Heckler \\ Department of Physics, The Ohio State University, 191 West Woodruff Ave, Columbus, OH 43210
}

\begin{abstract}
Introductory university physics frequently requires students to construct integrals. To explore students' abilities with this skill, we administered a simple physics problem requiring the construction of an integral to students enrolled in calculus-based introductory physics. Half of these students received written questions intended to scaffold construction of the integral, and the other half were not provided with scaffolding. Results indicate a strong interaction between scaffolding and student course grade. Specifically, students whose final class grade was above the median benefited significantly from the scaffolding, but students with grades below the median were not helped by the scaffolding. We also searched for other knowledge and skill factors that may inform instruction to improve integral construction skills, including spatial reasoning and conceptual understanding of the integral. Moderate, independent correlations with integral score were found for each, suggesting that instruction in these areas may positively influence performance.
\end{abstract}

Keywords: Integrals; Spatial Reasoning; Mass Density

PACS: $01.40 . \mathrm{Fk}$

\section{INTRODUCTION}

Integration is a common mathematical technique utilized in introductory university physics courses. While this skill is usually required learning in accompanying calculus courses, students may have trouble mapping their knowledge from mathematics to the context of physics to solve problems. Research conducted over the past few decades has shed light on this and other common difficulties many students face when attempting to solve integration problems in physics.

A common theme among the existing literature on this topic is a lack of fundamental understanding about the nature of an integral. Orton found that the majority of students (ages 16-22) in a British study did not recognize the integral as a limit of an infinite sum of products, or Riemann sum, though many were capable of computing the sum when asked [1]. Furthermore, Grundmeier et al. surveyed 52 students and found that their knowledge of the definition of the integral was independent of their ability to calculate its result [2]. They stated that students were able to "perform integration as a procedure with limited understanding that they are finding the area under the curve and that this area is being found as a limit of estimation for that area." Building on the work of Thompson and Silverman, who suggested that integration be emphasized in "layers," Nguyen and Rebello proposed four necessary steps to correctly use an integral in physics [3]. The steps include recognizing the need of an integral, correctly expressing the infinitesimal quantity to be summed, accumulating the infinitesimals, and computing the integral. In each step, they sought common difficulties among their student participants. They concluded that setting up the proper infinitesimal expression to be summed gave students significant trouble and sought to create tutorials to emphasize the meaning of the differential term (e.g., $d x, d \theta, d t, \ldots)$. In our present study, we found evidence that scaffolding within the problem similarly aimed at conceptual comprehension of the infinitesimal quantity may be ineffective for students whose physics course grade fell below the class median.

Another possible avenue of intervention to aid lowgrade students may be through spatial reasoning, such as the ability to mentally rotate $3 \mathrm{D}$ objects or carve $2 \mathrm{D}$ slices. Recent studies have found that spatial reasoning could be a primary obstacle to success in various STEM disciplines [4][5]. Moreover, correlation studies have determined that achieving success in some STEM fields is at least partially dependent on mental rotation and perspective taking [6][7][8].

In this study, we investigate how spatial reasoning ability and/or conceptual understanding may be tied to physics integration skills, particularly as a means of addressing the needs of low-grade students. We look for correlations and hierarchical relations between these skills to determine whether spatial or conceptual instruction might aid physics integration scores. 


\section{METHODS}

A total of 190 students participated in this study. All students were enrolled in the second semester of a calculus-based, introductory physics course at Ohio State University, a large public research institution. Students received course credit as part of their homework grade for participation in this assignment or an alternative assignment if they declined participation in the study. Over $95 \%$ of students in the class participated. Each student participant was instructed to complete a variety of computer and/or paper tasks in a quiet testing room for no more than 55 minutes. This particular study was conducted with written responses on paper.

Each student was given a short physics integral question, which featured a solid cylinder with a given mass density that varied along the length of the object (Fig. 1). The students were expected to setup but not compute an integral representing the total mass of the cylinder. In addition, a conceptual question (part a) asked each student whether the left third, middle third, right third, or none of the above would be heaviest if the cylinder were uniformly sliced into three smaller cylinders of equal length (called the "Heavy Slice" question).

Participants were randomly assigned to one of two conditions. The scaffolding condition received questions as shown in Figure 1, including part b, which steps the participants through a series of questions to (presumably) help them build the integral. The no scaffolding condition received an identical problem but were only given Parts $\mathrm{c}$ and a in that order. An integral score was computed to assess performance on Part c; students were given a maximum of $100 \%$ if they correctly included the integral boundaries $(25 \%)$, the cross sectional area of the cylinder (25\%), the length $d x$ of an individual slice of the cylinder (called the "differential;" 25\%), and the mass density of the cylinder $(25 \%)$. This integral score was graded holistically; for example, if any two of the four criteria were included, a score of $50 \%$ would be awarded.

Following the physics integration question, every student received a spatial reasoning test. This study used the Santa Barbara Solids Test (SBST)[9] for the first 123 students and the Purdue Spatial Visualization Test: Visualization of Rotations (PSVT:R)[10] for the final 67 students. Multiple spatial reasoning tests were utilized in order to ascertain possible correlations between physics integration and distinct spatial skills. The SBST requires students to mentally slice a 2D carving from a $3 \mathrm{D}$ object to produce a cross section. This is a skill that is conceptually similar to one utilized in the physics integral test in this study. The PSVT:R, by contrast, features a variety of 3D objects and requires students to mentally rotate each object to produce the correct orientation. The SBST was scored out of 29 possible correct answers and the PSVT:R was scored out of 30. A one-way ANOVA found no significant differences in final course grade between students who received the SBST and those who received the PSVT:R $(F(1)=0.656, p=0.419)$.

\section{RESULTS \& DISCUSSION}

\section{Effect Of Scaffolding}

A noteworthy quantity that guided the course of this study was the scaffolding condition integral score of students whose final physics grade fell below the class median (called "low-grade" students). A total of 41 low-grade students received the scaffolding condition; 20 of these students (48\%) had better than a $50 \%$ integral score. By contrast, 46 low-grade students received the no scaffolding condition, with 29 of them $(63 \%)$ scoring above a $50 \%$ on the integral question.

In a two-way ANOVA using both final course grade and integral test condition as independent factors on the integral score as the dependent variable, we (unsurprisingly) found a main effect that low-grade

A solid cylinder has radius $R$ and its central axis lies along the $x$-axis as shown, between $0 \leq x \leq l$. Both $x$ and $l$ are measured in $[\mathrm{m}]$ and the mass of the cylinder is measured in [kg]. Its mass density (per unit volume) is given by $\rho(x)=\rho_{0}\left(1-\frac{x}{2 l}\right)$.

a. Slicing the cylinder into three shorter cylinders each of equal length $\frac{l}{3}$, which (if any) piece is heaviest?

i. All pieces are equally heavy

ii. The left piece, from $0 \leq x \leq \frac{l}{3}$

iii. The middle piece, from $\frac{l}{3} \leq x \leq \frac{2 l}{3}$

iv. The right piece, from $\frac{2 l}{3} \leq x \leq l$

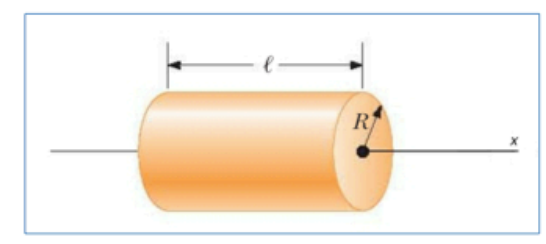

b. Now slice the cylinder into many thin circular layers, each with a width $d x$.

i. What are the units of $d x$ ?

ii. What is the cross-sectional area of each thin slice?

iii. What is the volume of each slice?

iv. Given that we can express Mass $=$ Density $\times$ Volume, what is the mass $d M$ of a thin slice located at the position $x$ ?

c. Write an integral expression (but do not solve) representing the total mass of the cylinder $M$. Clearly indicate the boundaries on the integral you provide.

FIGURE 1. Physics integration question including scaffolding questions b. i.-iv. 
students had lower integral scores than high grade students $\left(F(1)=9.075, p=0.003, \eta^{2}=0.047\right)$. However, we also found a significant interaction effect between course grade and integral test condition $(F(1)=6.683$, $\left.p=0.011, \eta^{2}=0.035\right)$. Specifically, low-grade students scored virtually the same as high-grade students in the no scaffolding condition ( $65 \%$ vs. $70 \%, d=0.25)$, but scored much lower than the high-grade students in the scaffolding condition ( $63 \%$ vs. $85 \%, d=0.81)$.

This interaction can be seen in Figure 2. The scaffolding increased the performance of high-grade students, but it had no (or possibly negative) effect on low-grade students. One possible explanation for this

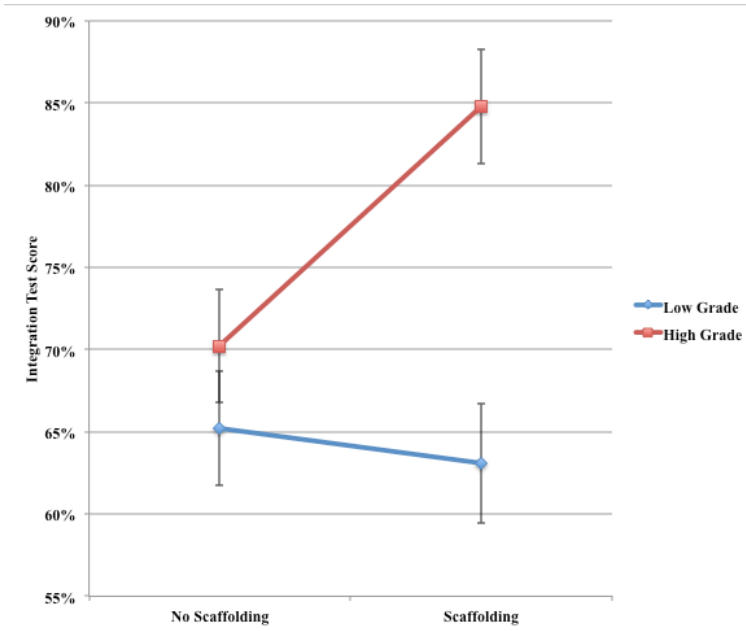

FIGURE 2. Integration score versus scaffolding conditions for both low- and high-grade students. Error bars are \pm 1 SE.

result is that scaffolding may be meaningful to students with deeper understanding of physics and mathematics, but for students who are struggling already, the extra layers of guidance may only confuse them and confound any prior understanding. This could affect the type of instruction needed to reach the low-grade students in order to improve their understanding and success in physics integrals. In the next section, we will further explore the conceptual understanding as a means of addressing the needs of low-grade students.

\section{Investigating The Influence Of Conceptual Understanding}

A possible pathway to integral score improvement is through conceptual understanding of the problem. The "Heavy Slice" question from the integral test (part a of Fig. 1) assesses a student's comprehension that the mass density varies, an important concept for understanding the meaning and necessity of integration in this task. While some students may have had trouble interpreting $\rho(x)$ and its direction of increase, we found that 90 of the 114 incorrect Heavy Slice responses simply invoked uniform density. If understanding (hence instruction) the variable density concept were necessary for accurate construction of the integral, we would expect that students with high integral scores would more frequently answer the Heavy Slice question correctly rather than incorrectly.

TABLE 1. Conceptual "Heavy Slice" Question crosstabulated with Integral Score. "Low" Integral Score implies $50 \%$ correct or below; "High" is greater than $50 \%$ correct. Both integral test conditions are combined. Both scaffolding conditions are combined.

\begin{tabular}{cccc}
\hline & & $\begin{array}{r}\text { Integral Score } \\
\text { Low High }\end{array}$ \\
\hline Low-Grade Students Only & & 31 & 25 \\
\cline { 2 - 4 } Heavy Slice Question & Right & 7 & 24 \\
\hline All Students & Wrong & 46 & 59 \\
\cline { 2 - 4 } Heavy Slice Question & Right & 14 & 61 \\
\hline
\end{tabular}

The results shown in Table 1 do not demonstrate this expected relationship. For low-grade students, we find that 25 participants obtained a high integral score while incorrectly answering the conceptual question, compared with 24 students earning a high integral score and correctly answering the conceptual question. This implies that a high score is attainable regardless of a student's understanding of the concept in the problem. This independence is seen across the entire sample of students (59 students answered the conceptual question incorrectly; 61 students answered it correctly; all earned a high integral score). Notably, students who correctly answered the conceptual question were far more likely to obtain an overall high integral score (24/31 for low-grade students; 61/75 for all students). Furthermore, students who incorrectly answered the integral question were very likely to answer the conceptual question incorrectly (31/38 for low-grade students; 46/60 for all students. These results imply that correctly setting up a physics integral may be a pre-requisite to understanding its meaning, rather than the reverse. This result seems to agree with the findings of Orton and Grundmeier et al. In sum, understanding the "heavy slice" concept is not necessary for correctly constructing an integral, but since a large proportion students who answer the concept question correctly also construct the integral correctly, it may be sufficient. Nonetheless, it is not clear that instruction on the "Heavy Slice" concept will improve performance on integration. Instead, these results indicate that such conceptual understanding is either hierarchically at a higher level than constructing an integral, or knowledge other than conceptual understanding is needed. 


\section{Investigating The Influence Of Spatial Reasoning}

Another possible avenue towards enhanced physics integration scores is spatial reasoning instruction. If correlations can be found between physics integration score and various spatial reasoning skills, it may suggest a hierarchy by which improved spatial reasoning leads to higher performance in integration.

The Santa Barbara Solids Test, administered to the first 123 student participants, demonstrated a weak correlation $(R=0.227)$ with the integral test score. Table 2 shows the cross-tabulation between SBST score and physics integral score. A "High" SBST score signifies the student performed higher than the median score ( $m e d=24$ out of 29 questions correct). These data do not demonstrate an obvious hierarchy of intervention. Because students who scored low on the SBST performed about equally poorly and well on the integral test, we find that the ability to produce mental cross sections of 3D objects may not be a pre-requisite to correctly setting up the physics integral from this study.

TABLE 2. Spatial reasoning scores cross-tabulated with Integral Score. "High" SBST/PSVT:R Score refers to the number of student scores above the median. "High" Integral Score implies greater than $50 \%$ correct. Both scaffolding conditions are combined.

\begin{tabular}{cccc}
\hline & & \multicolumn{2}{c}{ Integral Score } \\
& & Low & High \\
\hline \multirow{2}{*}{ SBST Score } & Low & 26 & 31 \\
\cline { 2 - 4 } & High & 11 & 23 \\
\hline \multirow{2}{*}{ PSVT:R Score } & Low & 11 & 17 \\
\cline { 2 - 4 } & High & 6 & 19 \\
\hline
\end{tabular}

Additionally, the Purdue Spatial Visualization (Rotations) Test provided a moderate correlation $(R=0.370)$ when compared with physics integral score. Table 2 shows a similar cross-tabulation between PSVT:R score and integration score. Once again, a "High" PSVT:R score is considered above the median (med $=25$ out of 30 questions correct). In spite of smaller statistics, a comparable pattern emerges again, wherein a student has a greater chance to obtain a high integral score even if the PSVT:R score is low. Likewise, students who score above the median on the PSVT:R are much more likely to score highly on the integration test than their peers who scored lower on the PSVT:R. This suggests that the ability to mentally rotate $3 \mathrm{D}$ objects may not be a pre-requisite to successfully setting up a physics integral.

\section{CONCLUSION}

This study found an important interaction: students whose final course grades fell below the median were clearly not helped by scaffolding questions intended to aid in the construction of a physics integral. By contrast, the high-grade students significantly benefitted from scaffolding on the integral construction task.

In order to determine possible avenues to enhance the physics integration ability of low-grade students, we also compared student performance on the integral task with performance on a conceptual/physical understanding task and standard spatial ability tasks. While there were correlations with performance on integrals, neither conceptual understanding nor spatial ability was found to be necessary: many students could correctly construct the integral but did poorly on conceptual understanding or spatial ability. Still, a large fraction of students who performed well on these tasks also did well on the integration task, and this suggests that instruction on these tasks might help performance on construction of an integral. Clearly, this is an empirical question that can only be answered in a controlled experiment via instructional intervention.

\section{REFERENCES}

1. A. Orton, Educ. Stud. Math. 14, 1-18 (1983).

2. T. Grundmeier, J. Hansen, and E. Sousa. PRIMUS 16, 178-191 (2006).

3. D. H. Nguyen and N. S. Rebello, Phys. Rev. ST Phys. Educ. Res. 7010112 (2011).

4. L. G. Humphreys, D. Lubinski, and G. Yao, J. App. Psy. 78, 250-261 (1993).

5. D. Lubinski, Personality and Individual Differences 49, 344-351 (2010).

6. M. Kozhevnikov, M. Hegarty, and R.E. Mayer, Diagrammatic representation and reasoning, 155171 (2002).

7. J. R. Pribyl and G. M. Bodner, J. Res. Sci. Teach. 24 229-240 (1987).

8. S. A. Sorby, Int. J. Sci. Educ., 31, 459-480 (2009).

9. C. A. Cohen and M. Hegarty, Proceed. $29^{\text {th }}$ Ann. Conf. Cog. Sci. Soc. 179-184 (2007).

10. S. Y. Yoon, Revised Purdue Spatial Visualization Test: Visualization of Rotations (Revised PSVT:R) (2011). 